\title{
Partitions with numbers in their gaps
}

by

\author{
Douglas Bowman (Urbana, Ill.)
}

1. Partitions with gaps. Bijections between various restricted partitions of integers have been extensively studied (see [2], [3]). In this paper we introduce a generalization of partitions, which are really a kind of restricted composition [3], and obtain bijections between certain classes of them and classes of ordinary partitions. Our generalization arises naturally in connection with solutions of $q$-difference equations and continued fractions. In fact, continued fractions provide an easy framework for analyzing these generalized partitions.

Throughout this paper we write an ordinary partition as

$$
n_{1}+\ldots+n_{k}, \quad n_{1} \geq \ldots \geq n_{k} .
$$

Define $n_{0}=\infty$ and $n_{k+1}=0$.

We consider the class $C(S, m)$ of partitions with parts $n_{i}$ taken from a set $S=\left\{a_{1}, a_{2}, \ldots\right\}$, where $a_{1}<a_{2}<\ldots$ We require that if $n_{i}=a_{r}$ and $n_{i+1}=a_{s}$ are two consecutive parts, then $r-s \geq m$. For such a partition we define the gapspace $g_{i}$ between $n_{i}$ and $n_{i+1}$ to be $r-s-m$. By convention we put $g_{0}=\infty$ and $g_{k}=t-m$, where $n_{k}=a_{t}$.

Before proceeding we give some examples of gapspaces.

Examples. 1. Consider partitions into parts which differ by at least 3; thus $S=\mathbb{Z}^{+}$and $m=3$. One such partition is $15+10+2$.

The gapspace between 10 and 2 is $(10-2)-3=5$ and the gapspace between 15 and 10 is $(15-10)-3=2$.

2. Consider partitions into non-consecutive odd integers; thus $a_{r}=2 r-1$ and $m=2$. One partition of this type is:

$$
63+51+17+13+7+1 .
$$

The gapspaces between the parts are:

$$
\infty, 4,15,0,1,1,-1 \text {. }
$$

Partially supported by National Science Foundation Grant DMS-9306089. 
We now introduce the class of generalized partitions to be studied in this paper. We start with partitions from the class $C(S, m)$. Note that such a partition can be written uniquely in the form

$$
a_{n_{1}}+a_{n_{2}}+\ldots+a_{n_{k}}, \quad n_{1}>_{m} n_{2}>_{m} \ldots>_{m} n_{k}
$$

where $x>_{m} y$ means that $x \geq y+m$. We now fix a positive integer $j$ and insert up to $g_{l}$ parts $j$ in the gap between $a_{n_{l}}$ and $a_{n_{l+1}}$. The resulting compositions are the objects of our study. Recall that two compositions are counted as distinct if their sequences of parts are distinct. Define $C(S, m, j)$ to be the class of compositions thus obtained. We refer to these compositions in picturesque language as "partitions with numbers in their gaps". We give some examples of partitions with ones in the gaps.

EXAMPLES. 1. We first consider $C\left(\mathbb{Z}^{+}, 2,1\right)$, i.e. partitions into natural numbers with minimal difference two with 1 's in the gaps. We start with a partition into parts with minimal difference 2 :

$$
21+19+12+7+1 \text {. }
$$

Then up to $g_{l}$ ones are inserted into the $l$ th gap; thus

$$
21+19+1+1+1+12+7+1
$$

and

$$
21+19+12+1+1+1+7+1
$$

and

$$
21+19+12+1+1+7+1+1
$$

are counted as distinct partitions of 63 . Another partition satisfying the conditions for this class of partitions is:

$$
\underbrace{1+\ldots+1}_{i \text { ones }}+21+19+12+7+1
$$

Recall that $g_{0}=\infty$, thus we may insert $i$ ones for any non-negative integer $i$.

It is easily found that the partitions of 3 of this type are:

$$
\begin{gathered}
3 \\
1+2 \\
1+1+1 .
\end{gathered}
$$

The partitions of 4 of this type are:

$$
\begin{gathered}
4 \\
3+1 \\
1+3 \\
1+1+2 \\
1+1+1+1,
\end{gathered}
$$


and the partitions of 5 are:

$$
\begin{gathered}
5 \\
4+1 \\
1+4 \\
1+3+1 \\
1+1+3 \\
1+1+1+2 \\
1+1+1+1+1 .
\end{gathered}
$$

Calling the number of partitions of $n$ of this type $P^{*}(n)$, it follows that $P^{*}(n)=3,5$, and 7 , for $n=3,4,5$.

2. Consider partitions into distinct non-consecutive odd numbers excluding 3 with ones in the gaps. Thus $m=2$ and

$$
a_{r}= \begin{cases}1 & \text { for } r=1 \\ 2 r+1 & \text { for } r>1\end{cases}
$$

To obtain the partitions of 11 in this class we first begin by forming partitions of numbers less than or equal to 11 into distinct non-consecutive odd numbers excluding 3 . These partitions are:

$$
\begin{gathered}
11 \\
9+1 \\
9 \\
7+1 \\
7 \\
5+1 \\
5 \\
1 .
\end{gathered}
$$

To these partitions we now insert up to $g_{l}$ ones into the $l$ th gap so that the sum of the resulting partition is 11 . This process yields:

$$
\begin{gathered}
11 \\
9+1+1 \\
1+9+1 \\
1+1+9 \\
1+1+1+7+1 \\
1+1+1+1+7 \\
1+1+1+1+1+1+5 \\
1+1+1+1+1+1+1+1+1+1+1 .
\end{gathered}
$$


Some of these partitions arise non-uniquely from our initial list of partitions. For example the partition $9+1+1$ arises from both the partition $9+1$ and the partition 9 , in the former case by inserting one 1 between the 9 and the 1, while in the latter case by inserting two 1's in the gap after the $9\left(g_{1}=2\right)$. Thus there are eight partitions of 11 in this example. The non-uniqueness problem could have been avoided by first listing just those partitions into parts greater than 1 and then inserting 1's according to the rule. The definition of gapspace assures that this would give the complete list of partitions of 11 we are considering.

We are now in a position to state some theorems. It may have been noticed in the first example that $P^{*}$ coincided with the ordinary partition function for the values considered. This is a consequence of the first theorem.

THEOREM 1. The number of partitions of $n$ into parts with minimal difference two with ones in the gaps is equal to $p(n)$.

A similar theorem giving a bijection with another well studied class of partitions is:

THEOREM 2. The number of partitions of $n$ into distinct non-consecutive odd parts $\left(a_{i}=2 i-1, m=2\right)$ with ones in the gaps is equal to the number of partitions of $n$ into distinct parts.

Corresponding to our second example we have

THEOREM 3. The number of partitions of $n$ into distinct non-consecutive odd parts excluding 3 with ones in the gaps is equal to the number of partitions of $n+1$ into an odd number of distinct parts.

In the theorems so far we had $m=2$. The following two theorems are for $m=1$.

THEOREM 4. The number of partitions of $n$ into distinct even parts with ones in the gaps is equal to the number of partitions of $n$ into parts satisfying $n_{1} \geq n_{2}>n_{3} \geq n_{4}>\ldots$

THEOREM 5. The number of partitions of $n$ into distinct odd parts with ones in the gaps is equal to the number of partitions of $n$ into parts satisfying $n_{1}>n_{2} \geq n_{3}>n_{4} \geq \ldots$

It is well known that the partitions in these last two theorems are equinumerous with partitions into parts $\equiv 1,2,5,6,8,9,11,12,14,15,18,19$ $(\bmod 20)$ and $\equiv 1,3,4,5,7,9,11,13,15,16,17,19(\bmod 20)$ respectively. See [6] and [7].

2. Proof of the theorems. In this section we give proofs of the last five theorems. In fact, they all are combinatorial interpretations of specializations of the $m=2$ case of the canonical $q$-difference equation for the 
general ${ }_{m} \Phi_{m-1}$. The following proposition is also used; it leads to partitions with parts in the gaps. It is interesting to note that partitions with numbers in their gaps give combinatorial interpretations of the partial quotients of two classes of continued fractions.

Proposition 1. Let $P_{n}$ and $P_{n}^{\prime}$ denote the $n$-th numerators of the convergents of the continued fractions

$$
\frac{P_{n}}{Q_{n}}=1+q^{j}+\frac{q^{a_{2}}-q^{j}}{1+q^{j}+} \frac{q^{a_{3}}-q^{j}}{1+q^{j}+\ldots} \frac{q^{a_{n+1}}-q^{j}}{1+q^{j}}
$$

and

$$
\frac{P_{n}^{\prime}}{Q_{n}^{\prime}}=1+q^{j}+q^{a_{1}}+\frac{-q^{j}}{1+q^{j}+q^{a_{2}}+\ldots} \frac{-q^{j}}{1+q^{j}+q^{a_{n+1}}} .
$$

Let $S=\left\{a_{2}, a_{3}, \ldots, a_{n+1}\right\}$ and $S^{\prime}=S \cup\left\{a_{1}\right\}$. Then

1. $P_{n}$ is the generating function for partitions from the set $C(S, 2, j)$, where the gapspace before the largest part $a_{k}$ is $n-k+1$.

2. $P_{n}^{\prime}$ is the generating function for partitions from the set $C\left(S^{\prime}, 1, j\right)$, where the gapspace before the largest part $a_{k}$ is $n-k+1$.

Proof. The standard recurrences for $P_{n}$ and $P_{n}^{\prime}$ are

$$
P_{n}=P_{n-1}+q^{j}\left(P_{n-1}-P_{n-2}\right)+q^{a_{n+1}} P_{n-2}
$$

and

$$
P_{n}^{\prime}=P_{n-1}^{\prime}+q^{a_{n+1}} P_{n-1}^{\prime}+q^{j}\left(P_{n-1}^{\prime}-P_{n-2}^{\prime}\right) .
$$

New partitions enumerated by $P_{n}$ are built up in the exponents of $q$ by adjoining $j$ or $a_{n+1}$ to the left of certain of those previously enumerated. The proof proceeds by induction. Let $A_{n}$ and $A_{n}^{\prime}$ be the generating functions for partitions of the types $C(S, 2, j)$ and $C\left(S^{\prime}, 1, j\right)$ respectively. It is easy to see that $P_{n}=A_{n}$ and $P_{n}^{\prime}=A_{n}^{\prime}$ for $n=0,1$. Assume $P_{i}=A_{i}$ and $P_{i}^{\prime}=A_{i}^{\prime}$ for $0 \leq i<n$. Now partitions enumerated by $A_{n}$ and $A_{n}^{\prime}$ fall into two classes:

1. those not containing the part $a_{n+1}$, and

2. those containing the part $a_{n+1}$.

This leads to two cases:

Case 1. Consider any partition $\pi$ enumerated by $A_{n}$ not containing the part $a_{n+1}$. Let $a_{k}$ be the part in $\pi$ with the largest subscript; so $k \leq n$. Now the gapspace before $a_{k}$ is $n-k+1$ by the definition of $A_{n}$. Suppose this gap is not full. Then by the induction hypothesis, $\pi$ is enumerated by $P_{n-1}$. On the other hand, if the gap is full, then the number of $j$ 's preceding $a_{k}$ is exactly $n-k+1$, and $\pi$ is not enumerated by the term $P_{n-1}$ in (1), again by induction and the definition of $A_{n}$. Then removing one of the $j$ 's leaves a partition $\pi^{\prime}$ with $n-k j$ 's preceding $a_{k}$. By induction $\pi^{\prime}$ is enumerated 
by $P_{n-1}$. Moreover, it has a full gap in $A_{n-1}=P_{n-1}$ and so as above is not enumerated by $P_{n-2}$. Hence $\pi$ is enumerated by the term $q^{j}\left(P_{n-1}-P_{n-2}\right)$ in (1). This case applies mutatis mutandis to the corresponding case for $A_{n}^{\prime}$ and $P_{n}^{\prime}$.

Case 2a. Consider any partition $\pi$ enumerated by $A_{n}$ containing the part $a_{n+1}$. Since $m=2$, the part with the next largest subscript is $a_{k}$ with $k<n$. Then the gapspace between $a_{n+1}$ and $a_{k}$ is $n-k-1$. Hence removing $a_{n+1}$ from $\pi$ leaves a partition $\pi^{\prime}$ beginning with at most $n-k-1$ $j$ 's followed by $a_{k}, k<n$. By induction $\pi^{\prime}$ is enumerated by $P_{n-2}$. Hence $\pi$ is enumerated by the term $q^{a_{n+1}} P_{n-2}$.

Case $2 \mathrm{~b}$. Consider any partition $\pi$ enumerated by $A_{n}^{\prime}$ containing the part $a_{n+1}$. Since $m=1$, the part with the next largest subscript is $a_{k}$ with $k \leq n$. Then the gapspace between $a_{n+1}$ and $a_{k}$ is $n-k$. Hence removing $a_{n+1}$ from $\pi$ leaves a partition $\pi^{\prime}$ beginning with at most $n-k j$ 's followed by $a_{k}, k \leq n$. By induction $\pi^{\prime}$ is enumerated by $P_{n-1}^{\prime}$. Hence $\pi$ is enumerated by the term $q^{a_{n+1}} P_{n-1}^{\prime}$. This completes the proof.

\section{Lemma 1. Suppose}

$$
c_{m} y_{m}=b_{m} y_{m+1}+a_{m} y_{m+2} \quad \text { for } m \geq 1 .
$$

Let

$$
\frac{P_{m}}{Q_{m}}=b_{1}+\frac{a_{1} c_{2}}{b_{2}+} \frac{a_{2} c_{3}}{b_{3}+\ldots} \frac{a_{m} c_{m+2}}{b_{m+2}},
$$

so that

$$
P_{k}=b_{k+1} P_{k-1}+a_{k} c_{k+1} P_{k-2} \quad \text { for } k \geq 1 .
$$

Then

$$
c_{1} c_{2} \ldots c_{n} y_{1}=P_{n-1} y_{n+1}+a_{n} P_{n-2} y_{n+2} \quad \text { for } n \geq 1 .
$$

Proof. When $n=1$ the lemma asserts that $c_{1} y_{1}=P_{0} y_{2}+a_{1} P_{-1} y_{3}$ $=b_{1} y_{2}+a_{1} y_{3}$, which is (3) with $m=1$. Assume the result true for $n=k$. Then the result for $n=k+1$ follows easily from the induction hypothesis, (3) and (4).

The $c_{m}=1$ case of this lemma is given in [8].

Proof of Theorems 1-5. As noted in [4] the sequences (see [5] for notation)

$$
\begin{gathered}
y_{m}={ }_{2} \Phi_{1}\left(\begin{array}{c}
-a q,-b q \\
c q^{2} ; x q^{m-1}
\end{array}\right), \quad a_{m}=a b x q^{m+1}-c q, \\
b_{m}=1+c q+(a+b) x q^{m}, \quad c_{m}=1-x q^{m-1}
\end{gathered}
$$


and the continued fraction

(7) $1+c q+(a+b) x q+\frac{\left(a b x q^{2}-c q\right)(1-x q)}{1+c q+(a+b) x q^{2}+\ldots} \frac{\left(a b x q^{n+1}-c q\right)\left(1-x q^{n}\right)}{1+c q+(a+b) x q^{n+1}}$

satisfy the conditions of Lemma 1 . Putting $a=b=0$ and $c=1$ in (6) gives

$$
\begin{gathered}
y_{m}=\sum_{n \geq 0} \frac{q^{(m-1) n} x^{n}}{\left(q^{2}\right)_{n}(q)_{n}}, \\
a_{m}=-q, \quad b_{m}=1+q, \quad c_{m}=1-x q^{m-1} .
\end{gathered}
$$

The continued fraction becomes

$$
1+q+\frac{x q^{2}-q}{1+q+} \frac{x q^{3}-q}{1+q+\ldots} \frac{x q^{n+1}-q}{1+q} .
$$

Let $P_{n}$ be the numerator convergent of this continued fraction. Equation (5) becomes

$$
(x q)_{n-1}(1-x) y_{1}=P_{n-1} y_{n+1}-q P_{n-2} y_{n+2} .
$$

Formally $\lim _{n \rightarrow \infty} y_{n}=1$. It is clear from the proof of Proposition 1 that as $n \rightarrow \infty, P_{n}$ formally tends to a limit which we call $P$. (The coefficient of $x^{m} q^{n}$ is the number of partitions of $n$ from $C\left(\mathbb{Z}^{+}, 2,1\right)$ containing $m$ parts greater than or equal to 2.) Thus letting $n \rightarrow \infty$ in (8) gives

$$
(x q)_{\infty}(1-x) y_{1}=(1-q) P .
$$

Notice that

$$
\lim _{x \rightarrow 1} c_{1} y_{1}=\lim _{x \rightarrow 1}(1-x) \sum_{n \geq 0} \frac{x^{n}}{\left(q^{2}\right)_{n}(q)_{n}}=\frac{1}{\left(q^{2}\right)_{\infty}(q)_{\infty}} .
$$

Letting $x \rightarrow 1$ in (9) gives

$$
(q)_{\infty} \frac{1}{\left(q^{2}\right)_{\infty}(q)_{\infty}}=(1-q) P
$$

where now $P$ is the generating function for $C\left(\mathbb{Z}^{+}, 2,1\right)$. Thus $P=1 /(q)_{\infty}$. It is well known that $1 /(q)_{\infty}$ is the generating function for ordinary partitions and so Theorem 1 is proved.

Theorem 2 is similar. Here $a=c=1$ and $b=-1$. Equations (6) become

$$
\begin{gathered}
y_{m}=\sum_{n \geq 0} \frac{(-q)_{n}}{(q)_{n}} q^{(m-1) n} x^{n}, \\
a_{m}=-x q^{m+1}-q, \quad b_{m}=1+q, \quad c_{m}=1-x q^{m-1} .
\end{gathered}
$$

The continued fraction becomes

$$
1+q+\frac{x^{2} q^{3}-q}{1+q+} \frac{x^{2} q^{5}-q}{1+q+\ldots} \frac{x^{2} q^{2 n+1}-q}{1+q} .
$$


Equation (5) becomes

$$
\left(1+x q^{m-1}\right) y_{m}=(1+q) y_{m+1}-\left(q+x q^{m+1}\right) y_{m+2} .
$$

Similarly,

$$
\lim _{x \rightarrow 1} c_{1} y_{1}=\frac{(-q)_{\infty}}{\left(q^{2}\right)_{\infty}} .
$$

The same reasoning as before shows that the limiting numerator convergent of the continued fraction

$$
1+q+\frac{q^{3}-q}{1+q+} \frac{q^{5}-q}{1+q+\ldots}
$$

is $(-q)_{\infty}$. Proposition 1 and the well known fact that this product is the generating function for partitions into distinct parts gives Theorem 2 .

To get Theorem 3, notice that by Theorem 3 of [4] the fact that the limiting numerator of the last continued fraction is $(-q)_{\infty}$ implies that the limiting denominator is

$$
\begin{aligned}
& \frac{(q)_{\infty}}{1-q}{ }_{2} \Phi_{1}\left(\begin{array}{c}
-q, q \\
q^{2}
\end{array} ; q\right)=\frac{(q)_{\infty}}{1-q} \sum_{n \geq 0} \frac{(-q)_{n}}{\left(q^{2}\right)_{n}} q^{n}=\frac{(q)_{\infty}}{2 q} \sum_{n \geq 0} \frac{(-1)_{n+1}}{\left(q^{2}\right)_{n+1}} q^{n+1} \\
& =\frac{(q)_{\infty}}{2 q}\left[\frac{(-q)_{\infty}}{(q)_{\infty}}-1\right]=\frac{1}{2 q}\left[(-q)_{\infty}-(q)_{\infty}\right] .
\end{aligned}
$$

On the other hand, the limiting denominator is equal to the limiting numerator of

$$
1+q+\frac{q^{5}-q}{1+q+} \frac{q^{7}-q}{1+q+\ldots} .
$$

By Proposition 1, Theorem 3 is proved.

To get Theorems 4 and 5 , put $q \rightarrow q^{k}, b \rightarrow q^{l-k} / x, c \rightarrow q^{j-k}$ and let $a, x \rightarrow 0$. Then equations (6) become

$$
\begin{gathered}
y_{m}=\sum_{n \geq 0} \frac{q^{k\left(\begin{array}{c}
n \\
2
\end{array}\right)+l n} q^{k(m-1) n}}{\left(q^{k+j} ; q^{k}\right)_{n}\left(q^{k} ; q^{k}\right)_{n}}, \\
a_{m}=-q^{j}, \quad b_{m}=1+q^{j}+q^{k(m-1)+l}, \quad c_{m}=1 .
\end{gathered}
$$

The continued fraction is then

$$
1+q^{j}+q^{l}+\frac{-q^{j}}{1+q^{j}+q^{k+l}+} \frac{-q^{j}}{1+q^{j}+q^{2 k+l}+\ldots} .
$$

Equation (5) becomes

$$
y_{1}=P_{n-1} y_{n+1}-q^{j} P_{n-2} y_{n+2} .
$$


By Proposition 1 , as $n \rightarrow \infty, P_{n} \rightarrow P$, where $P$ is the generating function for partitions from $C(S, 1, j)$ and $S=\{k n+l: n \geq 0\}$. So as $n \rightarrow \infty$, we get $y_{1}=\left(1-q^{j}\right) P$ from (10), which implies

$$
P=\sum_{n \geq 0} \frac{q^{k\left(\begin{array}{c}
n \\
2
\end{array}\right)+l n}}{\left(q^{j} ; q^{k}\right)_{n+1}\left(q^{k} ; q^{k}\right)_{n}} .
$$

When $k=2, j=1$ and $l=2,1$ respectively, the sum on the right generates partitions of the type required in Theorems 4 and 5 . This is not too hard to see directly, and is proved in [7].

In a future paper we shall give extensions of these theorems which also include not only the celebrated partition theorem of I. Schur (see [2], [9]) but also the generalization of it due to Alladi and Gordon [1].

\section{References}

[1] K. Alladi and B. Gordon, Generalizations of Schur's partition theorem, Manuscr. Math. 79 (1993), 113-126.

[2] G. E. Andrews, q-series: Their Development and Application in Analysis, Number Theory, Combinatorics, Physics, and Computer Algebra, CBMS Regional Conf. Ser. in Math. 66, Amer. Math. Soc., Providence, R.I., 1986.

[3] - , The Theory of Partitions, Encyclopedia Math. Appl., Vol. 2, Addison-Wesley, Reading, 1976. (Reissued: Cambridge University Press, London, 1985.)

[4] D. Bowman, Modified convergence for q-continued fractions defined by functional relations, in: Proc. Rademacher Centenary Conference, to appear.

[5] G. Gasper and M. Rahman, Basic Hypergeometric Series, Cambridge Univ. Press, 1990.

[6] B. Gordon, Some continued fractions of the Rogers-Ramanujan type, Duke Math. J. 32 (1965), 741-748.

[7] M. Hirschhorn, Developments in the theory of partitions, Dissertation, University of New South Wales, 1979.

[8] O. Perron, Die Lehre von den Kettenbrüchen, Vol. 2, Teubner, Stuttgart, 1957.

[9] I. Schur, Zur additiven Zahlentheorie, S.-B. Preuss. Akad. Wiss. Phys.-Math. Kl. 1926, 488-495. (Reprinted in I. Schur, Gessammelte Abhandlungen, Vol. 3, Springer, Berlin, 1973, 43-50.)

DEPARTMENT OF MATHEMATICS

UNIVERSITY OF ILLINOIS AT URBANA CHAMPAIGN

273 ALTGELD HALL, MC-382

1409 WEST GREEN STREET

URBANA, ILLINOIS 61801

U.S.A.

E-mail: BOWMAN@MATH.UIUC.EDU 